\title{
Mechanical and thermal stresses in a functionally graded rotating disk with variable thickness due to radially symmetry loads.
}

\begin{abstract}
Rotating disks have many applications in the aerospace industry such as gas turbines and gears. These disks normally work under thermo mechanical loads. Minimizing the weight of such components can help reduce the overall payload in aerospace industry. For this purpose, a rotating functionally graded (FG) disk with variable thickness under a steady temperature field is considered in this paper. Thermo elastic solutions and the weight of the disk are related to the material grading index and the geometry of the disk. It is found that a disk with parabolic or hyperbolic convergent thickness profile has smaller stresses and displacements compared to a uniform thickness disk. Maximum radial stress due to centrifugal load in the solid disk with parabolic thickness profile may not be at the center unlike uniform thickness disk. Functionally graded disk with variable thickness has smaller stresses due to thermal load compared to those with uniform thickness. It is seen that for a given value of grading index, the FG disk having concave thickness profile is the lightest in weight whereas the FG disk with uniform thickness profile is the heaviest. Also for any given thickness profile, the weight of the FG disk lies in between the weights of the all-metal and the all-ceramic disks.
\end{abstract}

Keyword: Rotating disk; Variable thickness; Thermoelasticity; Functionally graded material 